\title{
La séquence agonale comme procédé de la conflictualité médiatique
}

The agonal statement: a process of the media conflict

Nathalie Garric et Michel Goldberg

\section{(2) OpenEdition}

Journals

Édition électronique

URL : http://journals.openedition.org/tipa/1750

DOI : $10.4000 /$ tipa. 1750

ISSN : 2264-7082

Éditeur

Laboratoire Parole et Langage

Référence électronique

Nathalie Garric et Michel Goldberg, « La séquence agonale comme procédé de la conflictualité médiatique », TIPA. Travaux interdisciplinaires sur la parole et le langage [En ligne], 33 | 2017, mis en ligne le 09 octobre 2017, consulté le 25 septembre 2020. URL : http://journals.openedition.org/tipa/1750 ; DOI : https://doi.org/10.4000/tipa.1750

Ce document a été généré automatiquement le 25 septembre 2020.

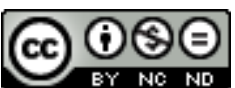

La revue TIPA. Travaux interdisciplinaires sur la parole et le langage est mise à disposition selon les termes de la licence Creative Commons Attribution - Pas d'Utilisation Commerciale - Pas de Modification 4.0 International. 


\section{La séquence agonale comme procédé de la conflictualité médiatique}

The agonal statement: a process of the media conflict

Nathalie Garric et Michel Goldberg

\section{Introduction}

1 Dans le cadre de cette contribution, nous nous intéressons à certaines modalités de construction de la communication verbale conflictuelle dans les espaces publics. Nous nous situons dans une analyse linguistique du discours pour étudier une controverse sociale à thème environnemental telle qu'elle s'expose dans le quotidien régional OuestFrance et telle qu'elle contribue à construire - amorcer, nourrir, désamorcer - un conflit inextricable et à mobiliser la population sur la question de l'extraction industrielle de sable en baie de Lannion.

2 Les conditions d'un espace délibératif de confrontation d'un discours et d'un contrediscours sont ici réunies pour la production d'une argumentation comprise comme « un mode de gestion discursive du désaccord » (Doury, 2016:22).

Argumenter un point de vue ne suppose pas que l'on cherche à se soustraire à la contradiction, mais que l'on cherche à voir jusqu'où une position peut « tenir » face à la contestation. Argumenter - même si ce n'est pas nécessairement l'objectif de tout locuteur développant un discours argumenté - permet de développer un point de vue au-delà de l'adhésion immédiate, d'en mesurer la cohérence, d'en tester la résistance, d'en expliciter les implications.

3 Mais alors que toute argumentation n'est pas systématiquement dominée par le conflit, il apparaît dans ce cas d'étude que la contradiction propre à l'argumentation prend systématiquement la forme d'une stigmatisation: tout au long de la lecture de notre corpus, nous sommes frappés par l'image pour le moins très négative de l'industriel qui 
a mis en place ce projet d'extraction (La Compagnie armoricaine de navigation, désormais CAN).

Face à ce discours argumentatif déséquilibré, et plus encore caractérisé par une prise de position polarisée (Amossy, 2014) susceptible de créer et de nourrir le conflit, nous nous sommes intéressés à la construction linguistique de cette image négative de l'un des contradicteurs (la CAN) pour étudier comment elle intervient dans l'espace public, en particulier dans la gestion sociale de la chose publique.

Cette contribution présente d'abord le contexte de la controverse et les objectifs de notre analyse. Elle se positionne ensuite dans le champ des recherches en analyse du discours et en argumentation qui ont donné lieu, notamment avec la parution de Polémiques médiatiques et journalistiques. Le discours polémique en question (Amossy \& Burger, 2011) et Apologie de la polémique (Amossy, 2014), à une abondante production sur la communication verbale plus ou moins violente (cf. Auger et al., 2008 ; Rosier, 2012). Enfin, elle se centre sur un procédé discursif récurrent, que nous proposons d'appeler procédé agonal, identifié comme symptôme de l'échange discursif conflictuel et plus généralement de la relation intersubjective conflictuelle. Son traitement s'appuie sur la notion de dialogisme (Bakhtine, 1975) telle qu'elle a été retravaillée notamment par Moirand (2007a, 2007b), mais également sur celle de sens commun telle qu'elle est développée par Sarfati dans le cadre de sa pragmatique topique (Sarfati, 2004 ; 2011). Nous étudions comment le discours peut être utilisé pour déplacer son équilibre argumentatif par la construction d'un ennemi, réceptacle des hostilités, dans le cadre d'un échange conflictuel monologué. Dans cet objectif, nous nous intéressons tout particulièrement à certaines marques linguistiques et discursives inscrites dans un "processus d'idéologisation » dans le sens où elles montrent un projet socio-discursif qui vise à construire une idéologie, c'est-à-dire un "discours mimétique à visée stratégique », caractérisé par une intention agonale et de l'ordre de l'« édiction d'une dogmatique » (Sarfati, 2011).

\section{Contexte et objectifs de l'analyse}

La Bretagne connaît depuis 2012 une controverse sociale à thème environnemental qui porte sur un projet industriel d'extraction de sable dans une dune en baie de Lannion. Elle confronte la CAN et de nombreux opposants (député, maires, élus, pêcheurs, plaisanciers, associations) à l'extraction de sable. Les partisans du projet mettent en avant des arguments liés à l'emploi, aux forts besoins en sable pour l'amendement des sols agricoles et à la nécessité actuelle d'importer ce sable. Les opposants mettent en avant des arguments liés à la protection de l'environnement côtier, au tourisme, à la pêche et à l'emploi.

7 Il ressort des discours produits dans le cadre de cette controverse une image négative, mais apparemment relativement consensuelle dans le paysage politique breton, de la CAN qui est en charge de l'extraction du sable : une société puissante, agissant dans les coulisses, ne dialoguant jamais sérieusement avec les groupes opposés au projet d'extraction de sable, prête à détériorer l'environnement et l'économie du littoral pour mener à bien ses objectifs financiers. Nous n'avons pas cherché quelle pouvait être la vérité des accusations et des représentations de cette société dans notre corpus, mais à recenser et à analyser les procédés linguistiques de cette disqualification systématique d'un des acteurs. Nous ne proposerons donc ici aucune lecture économique, 
sociologique ou environnementale de la controverse. Notre étude n'aborde pas la valeur des arguments, leur solidité, leur lien avec le réel. Notre étude n'a donc pas vocation à déterminer lequel des intervenants de la controverse « aurait raison».

En Bretagne, les discours en lien avec la question de l'extraction de sable sont nombreux et ils trouvent des dispositifs divers. Pour mener notre analyse, nous nous intéressons à l'un de ceux-ci: le discours médiatique régional défini comme espace institutionnel (voir infra Krieg-Planque \& Oger, 2010). Notre corpus est constitué de tous les articles parus dans Ouest-France entre le $1^{\text {er }}$ janvier et le 8 octobre 2015, comprenant le nom complet de la Compagnie armoricaine de navigation. Il a été constitué à partir de la base de recherche Factiva, portail d'articles de presse destiné à des institutions d'enseignement supérieur et à des entreprises. Il contient 58 articles parmi lesquels 57 articles pertinents parlent de la controverse liée à l'extraction de sable. La seule exception en est un article paru le 3 juillet, lequel traite de travaux de dragage à l'entrée d'un port et, de ce fait, celui-là est exclu de notre corpus. Parmi ces 57 articles, il s'en trouve une petite dizaine dont le thème principal n'est pas la controverse sur l'extraction de sable. Ils traitent cependant de cette extraction lorsqu'ils évoquent la CAN. C'est pourquoi ils sont maintenus dans notre corpus qui totalise donc 57 articles.

9 L'étude développée s'intègre dans un programme de recherche plus large qui porte sur deux domaines étroitement liés : (1) l'étude de l'argumentation dans des controverses sociales et environnementales (Garric \& Goldberg, 2012), et (2) la formation de jeunes scientifiques à l'étude des controverses sociales qui concernent leur discipline (Goldberg \& Crosse, 2013 : Goldberg, 2013 ; Goldberg \& Kraska, 2013). Elle s'inscrit dans l'école de tradition française de l'analyse du discours, notamment dans l'héritage de Pêcheux (1975), en privilégiant des outils d'analyse énonciative, pragmatique et argumentative pour favoriser une approche critique des controverses sociales à thème scientifique. Cette orientation critique adopte les Sciences du langage et en particulier l'analyse du discours pour appuyer son analyse d'objets discursifs engagés dans le débat public sur des observables langagiers précis. Ceux-ci doivent permettre d'aborder la violence verbale, le conflit discursif, à partir d'indices dont l'objectif est de comprendre et de faire comprendre la construction discursive de la controverse, en particulier la construction de l'ennemi dans un grand journal de la presse quotidienne dont l'équipe rédactionnelle adhère à une charte largement respectée. En effet, nous ne trouverons que fort rarement de propos injurieux, de lexique blessant, de stéréotype outrancier, d'attaques ad hominem dans notre corpus. Cependant, la nature conflictuelle de l'interaction n'en reste pas moins très forte. Il semble que le journal ne censure pas non plus les thèses de certains intervenants de la controverse : il cite, met en scène, les paroles d'intervenants appartenant aux deux «camps" principaux de la controverse. Leurs propos ne sont pas systématiquement déformés. Chaque thèse se fait entendre, de façon assez inégale cependant comme nous le verrons.

\section{Controverse, polémique, conflit}

Parlant de « controverse », nous jetons déjà un éclairage particulier sur notre corpus : il est alimenté par un désaccord que le discours médiatique, en raison de sa visée d'information, doit normalement donner à lire et à comprendre. Le désaccord ne saurait cependant être en soi conflictuel. Il est tout au plus problématique dans le sens où il renvoie à des positions contradictoires, mais a priori non inconciliables, autrement 
dit il n'exclut pas les points de vue alternatifs dans la recherche de solutions ou tout au moins d'explications. Il devient conflictuel par le mode de mise en relation de ses contradictions et par l'intentionnalité qui le sous-tend, notamment dès lors que le désaccord est entretenu et affirmé, qu'aucune solution n'est envisagée, et/ou qu'il est le plus souvent fidèle à la cause de l'un de ses contradicteurs par dichotomisation/ polarisation ou plus généralement par un processus d'idéologisation (Sarfati, 2011). Les deux notions de dichotomisation/polarisation sont introduites par Amossy, (2014:59) :

Cette dernière [la dichotomisation] exacerbe les oppositions jusqu'à les rendre inconciliables; elle relève d'une opération abstraite. La première [la polarisation] effectue des regroupements en camps adverses entre les participants, elle n'est pas d'ordre purement conceptuel, mais social.

Dans ces conditions, l'auteur utilise la notion de " polémique ", empruntée à Garand (1998 : 216) en relation avec celle de conflit :

Le dénominateur commun des énoncés polémiques en tous genres n'est donc pas la violence mais le conflictuel. Toute situation conflictuelle n'occasionne pas une prise de parole polémique (il y a des périodes où le conflictuel demeure latent, se résorbe, ne trouve pas d'issue dans les discours) mais, à coup sûr, toute parole polémique est issue du conflictuel.

12 La polémique est donc la manifestation verbale d'une situation conflictuelle qui se révèle par une " rhétorique du dissensus ", "c'est-à-dire [à] une gestion du conflit d'opinion sur le mode du dissentiment et non d'une quête de l'accord» (p. 42). Celle-ci est susceptible de cohabiter, de précéder ou de succéder à d'autres formes d'expression de la conflictualité, comme le conflit physique et/ou le conflit juridique (Garand, 1998). De la sorte, le conflit produit des discours contradictoires, dont la conflictualité verbale peut être la seule issue, mais qui sont également susceptibles d'ouvrir vers d'autres formes de conflictualité.

13 Dans ce qui suit, nous conservons la notion de polémique pour rendre compte du contexte d'étude dans lequel prennent place les extraits que nous analysons. Nous nous intéresserons plus singulièrement à certaines réalisations langagières qui mettent en jeu un procédé discursif commun, envisagé comme manifestation observable d'un conflit verbal, mais également comme forme susceptible de créer ou d'entretenir un conflit dans l'espace public sous quelque forme que ce soit. Nous posons que ces réalisations sont le point nodal de toute interaction conflictuelle qui ne saurait toutefois, comme tout discours, exister et être interprétée en dehors de l'ensemble des discours dans lequel elle prend place comme le soulignent Amossy et Burger $(2011: 15)$.

La polémique se réfère à un ensemble de discours qui circulent dans un espace social donné sur une question controversée, à laquelle sont données des réponses divergentes et mutuellement exclusives par des locuteurs qui tentent de disqualifier la thèse adverse ou l'adversaire qui la soutient. Elle comporte ses lieux, ses argumentaires et sa chronologie, tout en traversant les genres et les supports.

Pour autant, dans le cadre de cette contribution, notre réflexion est initiée à partir de séquences textuelles spécifiques qui témoignent d'une prise de position conflictuelle tout en contribuant à son élaboration. L'identification de cette séquence comme observable est construite en corpus et elle fonctionne comme indice de la polémique. Nous la désignerons en termes de séquence agonale. 


\section{La séquence agonale}

Une séquence agonale, que nous traitons comme une catégorie discursive, peut être un énoncé, un paragraphe ou un article complet. Cependant, nous nous intéresserons principalement ici aux énoncés agonaux parce qu'ils sont fréquents dans le corpus que nous analysons et qu'ils contribuent à l'effet de construction linguistique de l'ennemi que nous définissons comme caractéristique de l'interaction conflictuelle qui est au cœur de notre projet de recherche.

\subsection{Description de la séquence agonale}

L'énoncé agonal constitue la plus petite unité qui laisse entendre les personnages linguistiques nécessaires pour traiter par écrit d'une controverse: des acteurs qui s'opposent et un locuteur qui relate l'événement. Elle constitue de ce fait un indice de controverse. Lorsque des paroles ou des écrits de ces acteurs sont reprises dans les énoncés agonaux, ces acteurs peuvent être appelés " Proposant, Opposant, Tiers " comme le proposent les théories de l'argumentation (Plantin, 2016 : 525-529).

Parfois, l'énoncé agonal est le titre d'un article, ou le chapeau, ou l'intertitre. Parfois, il constitue le premier énoncé d'un article. Parfois, il est constitué d'un seul énoncé sans verbe.

18 Un procédé agonal présente une structure dont on distingue deux parties (dites P1 et P2). Le contenu de la première est une idée, une action, une information liée à un acteur 1 (A1) qui participe à la controverse. Le contenu de la seconde partie de l'énoncé exprime une opposition à la première partie et peut être attribué à un autre acteur 2 (A2) de la controverse.

1. Le futur comité de suivi censé rassurer... ne fait pas vraiment son effet pour l'instant sur les anti-extraction.

Partie $1(\mathrm{P} 1)$ : Le futur comité de suivi censé rassurer...

Partie 2 (P2) : ne fait pas vraiment son effet pour l'instant sur les anti-extraction

Verbe de l'énoncé (et adverbe rattaché) : ne fait pas vraiment

Acteur 1 (A1) : le ministère qui a mis en place ce comité de suivi

Acteur 2 (A2) : les opposants au projet d'extraction de sable

Locuteur : Sylvie Ribot, journaliste à Ouest-France.

Dans une séquence agonale, un contenu de $\mathrm{P} 1$, attribué à A1 est immédiatement contré dans P2 par A2, et c'est cette immédiateté des voix suggérées qui retient ici notre attention. L'énoncé donne à voir la position d'un intervenant sous le regard de son opposant, mais aussi sous le regard d'un locuteur (celui auquel est attribué le texte d'où est extrait l'énoncé agonal) qui organise les rôles. Dans cette mise en scène énonciative, P1 semble avoir pour fonction principale de donner à voir le point de vue de A2 au travers de $\mathrm{P} 2$, et de donner le dernier mot à $\mathrm{A} 2$. Très souvent dans notre corpus, c'est la CAN, ou le Ministère de l'Économie, qui sont entendus par la voix de A1, l'acteur dont le 
point de vue est systématiquement dévalorisé. Et ce sont des opposants au projet d'extraction (député, maire, pêcheur, plaisancier, etc.) qui sont entendus par la voix de A2. présentation de ces critiques : de ceux qui vivent de la mer». (28 septembre). controverse. pronom personnel elle désigne la CAN. : sa feuille de route un « carré des impossibilités ». (7 octobre). position critique émise par A2.

2. Celle, environnementale, sur laquelle sont sceptiques les opposants... et que contestent les pêcheurs, qui préféreraient un suivi espèce par espèce " (7 octobre). exclusivement l'opposition qu'il suscite. L'ensemble de l'article est consacré à la

3. Les élus estiment d'emblée que "M. Macron, le 14 septembre, a signé le décret accordant la concession de sables calcaires coquilliers à la Compagnie armoricaine de navigation, en dépit de l'avis unanime des collectivités, communes, communautés de communes, et des élus à tous les niveaux, malgré l'opposition massive de la population, au mépris des intérêts

Les séquences agonales peuvent donc servir d'amorce à un discours signalant au lecteur l'origine des critiques et des commentaires qui vont suivre. La parole de l'ennemi n'est entendue que pour permettre de situer le thème de la critique, de la condamnation, du jugement à l'encontre de son (ou de ses) adversaire(s). La séquence agonale sera ainsi souvent le premier (ou l'un des tout premiers) énoncé(s) d'une séquence plus ou moins longue dans laquelle s'expriment les thèses des opposants à un intervenant de la

L'opposition peut aussi s'exprimer sous la forme d'une mise en doute de la sincérité de l'ennemi ou de l'adversaire, comme dans cet exemple qui est constitué de la citation des propos d'une députée impliquée contre le projet d'extraction. Dans cet énoncé, le

4. Elle dit qu'elle va procéder à des sondages dès début 2016 mais tout en donnant déjà dans

La députée concentre ensuite son analyse à sa suspicion à l'encontre d'engagements pris par la CAN. Cette séquence agonale constitue donc à nouveau l'amorçage d'une

La séquence agonale peut aussi éclairer sur l'état d'esprit de A2 en réaction à un élément de la controverse initiée par A1 sans que nous sachions si ce ressenti a fait ou non l'objet d'une énonciation. Dans l'exemple suivant, il est question d'un comité de 
suivi chargé de veiller sur la protection du site au cours de la campagne d'extraction de sable :

5. Le futur comité de suivi censé rassurer... ne fait pas vraiment son effet pour l'instant sur les anti-extraction. (7 octobre).

La suite de l'article explicite l'opposition des anti-extraction sans jamais préciser le fonctionnement de ce comité de suivi, ni la position du ministre qui institue cette structure, ni sa position face aux critiques dont il fait l'objet.

La séquence agonale peut évoquer les sentiments mais également l'état d'esprit combatif des opposants pour accroître la suspicion à l'égard des faits évoqués dont le caractère ici instable qualifie la crédibilité du décret en question et de son acteur institutionnel :

6. Même si le décret, déjà quelque peu modifié, est tombé, pas question de lâcher prise sur l'extraction de sable par la CAN, compagnie armoricaine de navigation. (28 septembre)

Cette séquence est le premier énoncé de l'article et révèle le climat de la controverse tel qu'il s'y développe jusqu'à la fin.

\subsection{Fonctionnement dialogique du procédé agonal}

Ces différents extraits témoignent du dialogisme propre au discours (Bakhtine, 1975) tout en exploitant cette propriété constitutive (Authier-Revuz, 1982) à la dévalorisation systématique de l'un des acteurs de la controverse. Cette réalisation dialogique est dans notre corpus plus ou moins explicite: elle procède par des formes de discours rapportés (ex 3 et 4) ou par des formes d'hétérogénéité suggérée (Moirand, 2007a) qui fonctionnent par allusion à des dires ou des faits, par exemple en 4 et 5 , en faisant référence à l'intentionnalité de la CAN (« en donnant déjà dans sa feuille de route un "carré des impossibilités ») ou à celle du comité de suivi («le futur comité de suivi censé rassurer).

31 Nous identifions ainsi une forme d'hétérogénéité singulière que Moirand (2007a: 102) propose d'appeler interdiscursivité suggérée.

La particularité de ce qu'on appelle l'interdiscursivité suggérée permet au contraire d'inférer de signaux présents dans la matérialité textuelle la présence d'un interdiscours « déjà-là ", et donc de discours transverses. On repère par exemple la présence de substantifs désignant les séquences qui suivent ou qui précèdent comme des lieux de discours, ce qui du coup les caractérise comme des dires déjàdits [...]. On repère également la présence de séquences introduisant au fil du texte des dires qui sont attribués à d'autres ou à soi-même par d'autres, ce qui inscrit « en creux » l'opposition discursive entre deux camps adverses [...]. On repère enfin la présence de constructions favorisant l'inscription de positions antagonistes [...] qui laisse imaginer l'existence d'un contre-discours.

Ce dialogisme réalisé "sciemment" (nous faisons ici référence à la définition de l'interdiscours de Détrie, Siblot et Vérine, 2001: 155) est source de la conflictualité étudiée : il relève d'un dialogisme interdiscursif orienté systématiquement à l'encontre des défenseurs du projet d'extraction. En effet, partant de ce que «le dialogisme est analysable comme un dédoublement énonciatif» qui «pose les éléments non pas à égalité mais 
hiérarchiquement » (Détrie et al., 2001 : 85), nous posons qu'il contribue à la construction du sens en discours et, associé à d'autres marqueurs, à la construction du conflit.

Notre analyse s'appuie ici sur les travaux de Moirand qui propose d'articuler, malgré des origines théoriques différentes, les notions de dialogisme (Bakhtine, 1975) et d'interdiscours (Pêcheux, 1975). Ces deux notions renvoient communément à la présence d'une altérité dans le discours, mais leur articulation théorique vise à spécifier la nature de cette altérité. Elle peut se déployer selon Moirand (2004 : en ligne) sur deux axes du discours :

[...] les traces répertoriées dans le fil horizontal du discours (pour reprendre la métaphore de l'ADF) - ou de l'interaction - sont autant de lieux d'inscription de discours transverses renvoyant au fil vertical du discours. De ce fait, les observables repérés semblent avoir deux fonctions complémentaires: jouant un rôle dans l'orientation pragmatique des textes ou des interactions, et fonctionnant également comme autant de rappels mémoriels nécessaires à cette orientation.

Ces faits de dialogisme appliqués à notre exemple (1) consistent pour l'énonciateur effectuant les opérations modales dans cet énoncé en cours, à évaluer par la confrontation de $\mathrm{P} 1$ et de $\mathrm{P} 2$, et en l'occurrence par le marqueur évaluatif de réfutation "censé ", non pas le dictum de P1, mais l'énoncé antérieur rappelé par dialogisme interdiscursif tel qu'il a déjà fait l'objet d'opérations de modalisation. L'introduction dans P2 de l'adverbe "vraiment " tout en actualisant interdiscursivement la position des opposants déclenche simultanément un fait de dialogisme interlocutif qui anticipe la contestation possible de l'interlocuteur tout en tentant de l'orienter fermement vers le point de vue défendu.

La séquence agonale est une construction dialogique plurielle qui illustre une stratégie discursive, caractéristique de l'interaction conflictuelle. La modalisation de l'énoncé de A1 exprime la prétention du Ministère qui échoue dans sa tentative de rassurer et fonctionne comme un argument pour amener l'interlocuteur à un positionnement qui semble le plus raisonnable au regard du rapport dialogique mis en scène. Il convient effectivement d'insister sur l'inégalité énonciative produite par ces deux types de dialogisme. Brès et Mellet $(2009: 16)$ le rappellent :

Nous avons vu que le dialogisme interlocutif se construit sur l'interaction avec un énoncé prêté à l'allocutaire, à la différence du dialogisme interdiscursif qui se construit sur la présupposition d'un énoncé antérieur d'un tiers.

En d'autres termes, sous l'orchestration de la journaliste, le dialogisme interdiscursif démunit l'opposant de son statut d'interlocuteur pour le réduire à celui de tiers dont il est parlé et qui a disparu de l'actualité médiatique (son énoncé est évoqué), alors que le dialogisme interlocutif construit le lecteur du quotidien en interlocuteur contribuant à la connaissance actuelle de la question débattue. L'échange agonal est focalisé : la parole de A1 est rarement reprise du discours d'une personne ou d'une institution qui revendiquerait le propos de cet intervenant de la controverse. Cette parole n'est bien souvent connue que par la voix de A2. Elle est souvent mise à distance, critiquée, moquée par A2 et/ou par le locuteur. C'est une des raisons pour lesquelles il n'est pas toujours aisé de séparer les parties P1 et P2 d'un procédé agonal: le locuteur, qui privilégie le plus fréquemment le discours de A2 fait entendre sa voix lorsqu'il évoque la position de A1 dans P1.

37 Plus généralement, A1 est souvent peu entendu dans une séquence agonale : la partie P1 est souvent la plus brève, elle est même souvent beaucoup plus brève que P2. De plus, le cotexte est souvent silencieux au sujet de la position évoquée dans P1: le 
lecteur n'en saura donc pas plus sur la thèse de A1 en lisant tout l'article. Et lorsque ce cotexte n'est pas silencieux au sujet de la position de A1, il est soit très bref, soit très critique. À l'inverse, la position défendue dans P2 est souvent explicitée, soit dans la séquence agonale elle-même, soit dans le cotexte. Il arrive (rarement) que la séquence agonale accorde plus de place à l'énonciation de A1 que de A2 :

7. Quand le ministère de l'Économie a accordé il y a dix jours le titre minier à la Compagnie armoricaine de navigation (CAN) pour extraire du sable en baie de Lannion, la grande majorité de la population a vu rouge. (25 avril)

Cependant, le cotexte de cet article est tout entier dédié à la thèse de A2 (c'est-à-dire aux opposants au projet).

A1 est souvent désigné par un lexique " froid » (la CAN, l'industriel, le ministre). À l'inverse, A2 est souvent désigné par le nom d'une personne, d'une profession (les pêcheurs, etc.). Ces désignations peuvent créer un effet quelque peu déshumanisé pour A1 et humanisé pour A2. Cette présentation inégale des acteurs et des énonciateurs contribue à une construction d'un ethos inégal de chacun des énonciateurs, l'un d'eux, stigmatisé, devient le pôle faible du conflit. Il peut dès lors devenir la cible de la suite de l'article journalistique.

Pour illustrer ce fait, nous proposons ici un tableau qui reprend les différentes désignations des intervenants dans l'article paru le 7 octobre.

\begin{tabular}{|l|l|}
\hline Désignation de la CAN et de ses représentants & Désignation des opposants \\
\hline & pêcheurs \\
& La députée PS Corinne Erhel \\
Compagnie armoricaine de navigation (CAN) & L'élue \\
CAN, filiale du groupe Roullier & Le collectif Peuple des dunes \\
& Lannion Trégor communauté \\
& Les anti-extraction \\
& Alain Bidal, président de Peuple des dunes \\
\hline
\end{tabular}

41 Le procédé agonal est donc une structure qui peut être apparemment relativement simple, mais son analyse est complexe: trois personnages (trois acteurs socioidéologiques, trois rôles argumentatifs, trois énonciateurs) et un contexte interdiscursif spécifique.

Cette complexité interdiscursive est aussi une source d'informations importante pour l'analyse : en effet, ce type d'énoncé synthétique peut souvent représenter le "climat " de l'ensemble d'un article, orienter les choix rédactionnels du locuteur et par là, potentiellement exprimer la position des différents acteurs socio-politiques dans le règlement de la question publique. Le dialogisme interdiscursif donne plus encore une teneur foncièrement conflictuelle au moment discursif, notamment s'il est combiné à d'autres opérations caractéristiques de la polémique et au service d'une stratégie d'idéologisation. C'est à ce fonctionnement discursif que nous nous consacrons dans ce qui suit. 


\section{Fonctionnement discursif de la séquence agonale} deux courts paragraphes). De par leur brièveté, elles peuvent, dans certains cas, être les révélateurs d'une stratégie d'écriture qui confine au simplisme, au jugement peu informé, au suivisme face à une opinion publique ou face à des groupes déterminés, et enfin au manichéisme qui désigne une fois pour toutes les bons et les mauvais dans une controverse. Nous formulons l'hypothèse que, par ces propriétés, ils réalisent un processus d'idéologisation servant le positionnement des opposants.

\subsection{Pragmatique topique et processus d'idéologisation}

La pragmatique topique élaborée par Georges-Elia Sarfati (2011) développe une conception socio-discursive de l'activité linguistique de tout locuteur. En ce sens, elle est susceptible de fournir un cadre théorique à l'analyste de discours qui associe son activité scientifique à une visée implicative ou critique. En traitant de la notion de conflit dans l'espace médiatique, nous adoptons cette visée : le journaliste intervient dans l'espace public afin de proposer des éléments d'interprétation et de compréhension de questions sociales plus ou moins problématiques. Ce traitement suscite un enjeu éthique dans le sens où il permettra au citoyen de participer à sa manière au débat public. Il convient donc d'analyser comment le conflit contribue à la visée d'information citoyenne démocratique.

Du point de vue de la pragmatique topique, les discours s'entendent comme production de sens commun, ce dernier étant ainsi défini par Sarfati $(2011: 145)$.

Ensemble des manières de signifier et des savoirs propres aux membres d'une même communauté de sens, en tant qu'ils sont investis dans une même relation d'objet, en vue de fins spécifiques, internes et externes à cette communauté.

Toute institution de sens (le discours médiatique par exemple) est animée par l'activité linguistique de ses communautés de sens (les partisans et les opposants par exemple). Elles produisent et construisent leur sens commun, à partir d'une topique directionnelle et d'une dynamique qui leur sont propres, selon les modes de variation prédictibles et descriptibles. L'analyse du sens commun doit permettre ainsi d'analyser les motifs qui orientent les sujets vers certaines formes de sémiotisation dans tel ou tel type de pratiques sociales. Les dispositifs normatifs du sens commun trouvent trois types de mise en discours : dans une visée fondatrice et instituante, ils procèdent par des énoncés canoniques, dans une visée de transmission et d'exposition plus ou moins large, ils procèdent par des énoncés de la vulgate, enfin dans une visée de banalisation et d'évidence, ils procèdent par des énoncés doxiques. La doxa est l'état résultant de ces topoi depuis leur source canonique dont elle dérive comme "naturellement " en passant par différentes formes de vulgate. La doxa se distingue de l'idéologie et chacune correspond à deux états discursifs distincts. L'idéologie est obtenue par un processus d'idéologisation. Autrement dit, pour Sarfati (2011), il n'existe pas de discours

TIPA. Travaux interdisciplinaires sur la parole et le langage, 33 | 2017 
idéologique a priori mais seulement des usages idéologiques des discours par un processus d'idéologisation qui sert la visée stratégique d'une communauté de sens.

La formation d'une doxa consiste [...] dans un processus d'entropie spontanée, indissociable de l'activité verbale des sujets-acteurs en rapport avec l'institution de sens dont elle est issue [...]. Mais la formation d'une doxa peut aussi résulter d'une intention ou d'un projet socio-discursif précis [...] qui ne relève pas d'un phénomène d'entropie, mais à l'inverse d'une détermination ainsi que d'une définition délibérée, autrement dit de l'édiction d'une dogmatique (Sarfati, 2011: 154).

Par ce second mode de formation, la doxa devient alors idéologie. Sa finalité est planifiée et réfléchie, son point de vue est réducteur et orienté ou axiologisé selon la distinction ami/ennemi ${ }^{1}$, son intention est agonale et son mode de diffusion est hégémonique. L'auteur souligne enfin que l'idéologisation « constitue un lieu privilégié de mise en œuvre de la polyphonie ».

\subsection{Dichotomisation et polarisation}

Nous reconnaissons ainsi dans ce processus de nombreuses propriétés accordées aux séquences agonales, mais également à la polémique telle qu'elle est définie par Amossy (2014). Les opérations de dichotomisation et de polarisation peuvent effectivement répondre directement aux caractéristiques de l'idéologisation. Dans nos exemples, elle prend la forme de deux camps adverses, les opposants et les partisans à l'extraction du sable en baie de Lannion avec une valorisation des opposants qui minimise, voire dévalorise, les partisans.

Dans l'exemple suivant, où la séquence agonale se développe sur deux paragraphes, la polarisation est très explicitement identifiable. Comme illustré en (8), P2 est souvent constitué d'une citation de $\mathrm{A} 2$, alors que $\mathrm{P} 1$ n'est que très rarement (voire jamais) une citation :

8. Emmanuel Macron, le ministre de l'Économie, a accordé mardi à la Compagnie armoricaine de navigation (CAN, groupe Roullierde Saint-Malo, 7000 salariés) une concession d'extraction de sable coquillier en baie de Lannion, pour une année, reconductible.

Cette décision passe très mal auprès des gens de mer et des défenseurs de l'environnement. «À la première extraction, le mal sera fait et les conséquences irréversibles, s'indigne Philippe Priser, marin-pêcheur ligneur dans la baie de Lannion. C'est beaucoup plus facile de faire fuir le poisson que de le faire revenir. On ne peut pas admettre qu'un privé vienne piller la mer et notre outil de travail. » (19 avril 2015)

51 Les deux camps apparaissent, ils correspondent aux deux seuls paragraphes structurant l'article. On trouve d'une part, sous une forme condensée, la compagnie d'extraction évoquée par l'intermédiaire de l'accord du Ministre de l'économie et dont seul l'état civil et juridique est mentionné ; et d'autre part, l'énumération de discours rapportés d'un témoin opposé à l'extraction et qui focalise sa critique sur une dimension environnementale qui entretient un «localisme breton». Ces propos sont présentés comme ceux du plus grand nombre (on opposera le déterminant défini pluriel «les» au déterminant singulier "la») et à l'aide de structures génériques (structure présentative et emploi du pronom « on ») qui reproduisent la doxa. Par des 
procédés de «montée en généralité » (Doury \& Lefébure, 2006: 60), l'enjeu du traitement journalistique devient alors autre.

D'une part, il y a généralisation sur le fond dans un effort que l'orateur produit sur lui-même pour développer, avec le souci de la validité interne, l'intelligibilité de l'enjeu discuté. D'autre part et conjointement, il y a invocation d'identité(s) sociale(s) partagée(s) dans un effort d'implication externe par lequel l'orateur situe l'enjeu non pas seulement en tant que tel mais relativement à une ou des collectivités humaines.

Cette montée en généralité est particulièrement illustrée par l'exemple (3) dans lequel le poids accordé à chacune des parties est fortement inégal. Alors que la position d'E. Macron n'est qu'évoquée par l'intermédiaire du décret mentionné, les opposants sont actualisés par un concert de voix qui semblent occuper tout l'espace.

Les élus estiment d'emblée que «M. Macron, le 14 septembre, a signé le décret accordant la concession de sables calcaires coquilliers à la Compagnie armoricaine de navigation,

a) en dépit de l'avis unanime des collectivités, communes, communautés de communes, et des élus à tous les niveaux,

b) malgré l'opposition massive de la population,

c) au mépris des intérêts de ceux qui vivent de la mer ». (28 septembre)

Les séquences a), b) et c) formulent trois contre-discours dont l'accumulation marque une opposition massive, renforcée par la variation de la locution prépositionnelle ( $«$ en dépit de ", " malgré », " au mépris de ») qui permet de répéter la valeur oppositive, par la caractérisation quantifiante des voix évoquées ("avis unanime», "opposition massive ») et par la détermination générique des syntagmes désignant les opposants.

Cette montée en généralité s'inscrit dans la définition d'un consensus avec le lectorat qui devient dès lors partie prenante du conflit et de son orientation. Autrement dit, nous assistons à un processus qui accorde au citoyen local le statut d'allié des opposants, ceux-ci ne pouvant se poser contre la cause défendue, la défense de l'environnement ou la préservation d'un site particulier par exemple. Le procédé agonal ouvre alors à la possible critique tous azimuts de la position de partisans : le conflit est nourri et inégal, il en vient à encourager d'autres formes de manifestations conflictuelles :

9. Le Premier ministre et le ministre de l'Économie Emmanuel Macron ont signé et fait paraître le décret autorisant la concession demandée par la CAN (permis minier) en vue de l'extraction de sable coquillier en baie de Lannion. "Cet arrêté ministériel sera attaqué et des requêtes, recours en annulation, auprès du Conseil d'État seront déposées par les opposants se constituant partie civile et ayant juridiquement intérêt à agir. » (25 septembre)

10. Après la signature du décret autorisant l'extraction de sable par la Compagnie Armoricaine de Navigation, l'association «Pleumeur-Bodou nature » invite la population et les élus à un rassemblement devant la mairie de Pleumeur-Bodou. (17 septembre)

\section{Réflexion sur une conflictualité institutionnelle}

Nous ne nous sommes pas jusqu'ici intéressés aux dimensions institutionnelle et médiatique de la controverse étudiée. Pourtant la conflictualité exprimée, notamment 
par les séquences agonales, invite à quelques réflexions. En effet, les discours institutionnels sont habituellement définis, en référence aux travaux de Krieg-Planque et Oger (2010: 93), par deux propriétés.

- la stabilisation des énoncés : « la production des discours institutionnels, tout autant que la circulation des énoncés dans l'espace public, obéissent à des régularités qui réduisent la diversité des énoncés possibles ».

- l'effacement de la conflictualité : «dans l'institution, les locuteurs, sommés de répondre à des exigences complexes voire contradictoires, obéissent à une injonction plus générale : organiser la polyphonie, réduire la dissonance, produire la cohérence ».

Le caractère institutionnel d'un discours est un mode de donation ou d'exposition des discours dans l'espace public. Dans ce cadre normé, le rôle du discours médiatique est d'informer le citoyen par une activité de révélation, voire d'élucidation. Autrement dit, en tant qu'institution, les médias se doivent de rapporter une information interprétée qui s'établit dans un certain consensus avec une mise à disposition plus ou moins grande du cheminement vers la conclusion exposée. Ainsi est-il construit l'effacement de la conflictualité nécessaire à son fonctionnement institutionnel (Garric, à paraître).

Nous défendons en outre l'hypothèse selon laquelle lorsque les médias ne peuvent maintenir cet objectif, ils accompagnent leur discours de commentaires métalangagiers qui glosent leur traitement. Nous avons formulé l'hypothèse selon laquelle dans le contexte notamment de traitements à caractère scientifique et sanitaire, consacrés par exemple à l'énergie nucléaire, aux OGM, aux nanotechnologies ou encore au réchauffement climatique, où les journalistes sont privés du consensus scientifique par la publicisation de l'objet scientifique et de la polémicité interne du discours scientifique, ils ne peuvent plus jouer leur rôle de vecteur institutionnel dans l'espace du débat public. Ils s'expriment alors sous la forme de commentaires métadiscursifs qui formulent progressivement un constat presque unanime: les questions scientifiques abordées relèvent du " débat impossible » et, plus encore, de la "polémique ». Ces deux termes d'abord occurrents dans le corps des articles entrent progressivement dans le titrage des quotidiens et cadrent la lecture des traitements ${ }^{2}$ (Garric, à paraître). Or, de façon surprenante, le traitement médiatique de la controverse étudiée ne respecte pas ces données génériques. Si une forme de consensus naît autour de la cause défendue par les opposants, celle-ci est construite par le biais d'un processus d'idéologisation souvent péjoratif - qui laisse s'exprimer la conflictualité. La manifestation de celle-ci est d'autant plus forte qu'elle s'accompagne de la construction du rôle de l'ennemi.

Nous formulons l'hypothèse selon laquelle ce développement marginal dans le discours médiatique est propre au statut de presse régionale de notre support. La controverse a très peu intéressé la presse nationale, elle est d'ailleurs peu connue sur le territoire national. En revanche, en Bretagne, elle se révèle particulièrement présente d'une part, extrêmement virulente d'autre part, au point de susciter la crainte de formes de conflit physique. La proximité du média, de l'objet discursif (dune de sable en baie de Lannion) et du lectorat pourraient donc constituer un point de rupture, et probablement d'affaiblissement de sa dimension institutionnelle, avec les contraintes génériques du discours médiatique non régional. 


\section{Conclusion}

L'analyse du conflit langagier ne peut se suffire d'une approche syntaxique et lexicale. Il apparaît en effet que le conflit ne procède pas par des marqueurs locaux de la violence verbale, mais par un procédé discursif dialogique qui prend sens dans l'interdiscursivité d'un moment discursif. Le conflit langagier étudié non seulement témoignerait de l'existence d'un conflit socio-idéologique préalable, mais pourrait également se présenter comme un moyen de contenir autant que possible des manifestations conflictuelles autres, notamment physiques. Cependant, en tout état de cause, comme en témoigne la fréquence du procédé agonal, le discours médiatique régional, pour tenir cet objectif apparemment pacifiste, entretient le conflit verbal en tentant d'imposer par le processus d'idéologisation et la primauté accordée à l'une des voix de la controverse, les opposants, une nouvelle forme de doxa à partir d'une idéologie.

[...] si une doxa peut servir de base à l'élaboration d'une idéologie, il est presque exclu qu'une idéologie donne lieu à une doxa [...], sauf à considérer que l'usage idéologique d'un discours constitue une nouvelle forme canonique, auquel cas il faut envisager, dans une même formation sociale, que deux versions d'un même discours entrent en concurrence, chacun produisant son « canon ", sa " vulgate » et sa «doxa», mais à partir de types de sémiotisations et finalités radicalement distinctes (Sarfati, $2011:$ 155-156).

60 En termes d'analyse du discours à visée pédagogique ou plus largement critique, aiguillée par l'occurrence de séquences agonales, il est souvent intéressant de relever certaines autres caractéristiques de l'énoncé :

- l'énoncé agonal peut être un révélateur de l'existence d'une controverse ;

- l'énoncé agonal peut être un révélateur du « climat » de la controverse ;

- le lexique utilisé pour désigner A1 et A2, ainsi que la position qui leur est attribuée dans P1 et P2, peuvent être constitutifs d'une construction linguistique de l'ennemi. Par exemple, ce sera le cas si les termes qui désignent l'un des acteurs sont péjoratifs ;

- la prise de distance ou, au contraire, les marques de proximité entre le locuteur et chacun des deux acteurs A1 et A2, peuvent nous indiquer si un journaliste ou la rédaction d'un journal prend position dans une controverse ;

- de nombreuses autres marques linguistiques d'un procédé agonal peuvent contribuer à la construction linguistique de l'ennemi (implicite, humour, marques d'atténuation ou de renforcement, paroles rapportées, etc.).

61 Le cotexte de l'énoncé agonal éclaire aussi les choix rédactionnels du locuteur, notamment dans sa construction de l'ennemi. Par exemple, il peut se révéler intéressant de vérifier si le cotexte est silencieux au sujet de A1 et de sa position, ou s'il renforce le message de A2. Dans ces deux cas, on peut se demander si A1 est véritablement entendu dans l'ensemble du texte ou du corpus, s'il n'est pas systématiquement l'objet d'attaques ou de condamnations. 


\section{BIBLIOGRAPHIE}

Amossy, R. (2014) Apologie de la polémique, Paris: PUF (Interrogation philosophique).

Amossy, R. \& M. Burger (2011) Introduction : la polémique médiatisée, Semen [en ligne], 31. Disponible sur : http://semen.revues.org/9072 (consulté le 24 janvier 2016).

Auger, N., Fracchiolla B., Moïse, C. \& C. Schultz-Romain (2008) De la violence verbale pour une sociolinguistique des discours et des interactions [en ligne], in Congrès Mondial de Linguistique Française, 9-12 juillet 2008, Paris, France. Disponible sur : https://doi.org/10.1051/cmlf08140 (consulté le 20 juin 2017).

Authier-Revuz, J. (1982) Hétérogénéité montrée et hétérogénéité énonciative : éléments pour une approche de l'autre dans le discours, DRLAV, Revue de linguistique, 26, p. 91-151.

Bakhtine, M. (1975) Esthétique et théorie du Roman, Paris : Gallimard (Tel).

Brès, J. \& S. Mellet (2009) Une approche dialogique des faits grammaticaux, Langue française, 3, 163, p. 3-20.

Détrie, C., Siblot, P. \& B. Verine (2001) Termes et concepts pour l'analyse du discours. Une approche praxématique, Paris: Honoré Champion.

Doury, M. (2016) Argumentation. Analyser textes et discours, Paris: Armand Colin.

Doury, M. \& P. Lefébure (2006) «Intérêt Général », « Intérêts Particuliers », Questions de communication, 9, p. 47-71.

Garand, D. (1998) Propositions méthodologiques pour l'étude du polémique, États du polémique, p. 211-268.

Krieg-Planque, A. \& C. Oger (2010) Discours institutionnels : perspectives pour les sciences de la communication, Mots. Les langages du politique, 94, Lyon: ENS Éditions, p. 91-96.

Garric N. (2017) Homogénéisation des discours institutionnels : le cas de l'objet scientifique sanitaire, Le Discours et la Langue. Revue de linguistique française et d'analyse du discours, 9.1, Louvainla-Neuve: EME Editions, p. 31-48.

Garric N. \& M. Goldberg (2012) Mise en scène de la scientificité dans le débat citoyen. Le cas des OGM comme argument d'une lettre ouverte autour de la science, Scientisme(s) \& communication, Paris: L'Harmattan, p. 159-168.

Goldberg, M. \& M. Crosse (2015) Étudier les controverses en Faculté des Sciences et en enseignement à distance. La pensée critique et les outils de l'analyse de discours, in Actes du colloque international « Questions de pédagogies dans l'enseignement supérieur », Brest, p. 911-916.

Goldberg, M. (2013) Pensée critique à l'université dans des formations professionnalisantes. Une étude sur des aptitudes des étudiants pour repérer des arguments fallacieux dans des controverses, in Actes du colloque international «Questions de pédagogies dans l'enseignement supérieur ", 4-6 juin, Sherbrooke, Canada, p. 745-755.

Goldberg, M. \& G. Kraska (2013) Un travail collaboratif en contrôle continu avec un grand nombre d'étudiants, in Actes du colloque international "Questions de pédagogies dans l'enseignement supérieur ", 4-6 juin, Sherbrooke, Canada, p. 114-122. 
Maldidier, D. (1993) L'inquiétude du discours. Un trajet dans l'histoire de l'analyse du discours : le travail de Michel Pêcheux, Semen [en ligne], 8. Disponible sur : http://semen.revues.org/4351 (consulté le 11 août 2016).

Moirand, S. (2004) Le dialogisme, entre problématiques énonciatives et théories discursives, Cahiers de praxématique [en ligne], 43. Disponible sur : http://praxematique.revues.org/1853 (consulté le 16 décembre 2016).

Moirand, S. (2007a) Les discours de la presse quotidienne. Observer, analyser, comprendre, Paris: PUF (Linguistique nouvelle).

Moirand, S. (2007b) Discours, mémoires et contextes : à propos du fonctionnement de l'allusion dans la presse, Corela [en ligne], HS-6. Disponible sur : https://doi.org/10.4000/corela.1567 (consulté le 10 décembre 2016).

Pêcheux, M. (1975) Les Vérités de La Palice, Paris: Maspero.

Plantin, C. (2016) Dictionnaire d'argumentation. Une introduction aux études d'argumentation, Lyon: ENS Editions.

Rosier, L. (dir.) (2012) Insulte, violence verbale, argumentation, ADARR [en ligne], 8. Disponible sur : https://aad.revues.org/1242 (consulté le 13 juin 2017).

Sarfati, G-E. (2011) Analyse du discours et sens commun : institutions de sens, communautés de sens, doxa, idéologie, in Matériaux philosophiques pour l'analyse du discours, Besançon: Presses Universitaires de Franche-Comté, p. 139-173.

Sarfati, G-E. (2004) La sémantique : de l'énonciation au sens commun. Éléments d'une pragmatique topique, Texto! [en ligne], décembre 2004. Disponible sur : http://www.revuetexto.net/Inedits/Sarfati/Sarfati_Semantique.html (consulté le 16 février 2016).

\section{NOTES}

1. L'auteur précise que «[c]ette distinction fondamentale commande ici l'ordre du discours; selon les situations, elle s'entend également avec davantage de nuance, sur le mode de la distinction : soi et les autres (alliés, partenaires, concurrent, adversaire, etc.) (Sarfati, 2011 : 158 ). 2. Par exemple : OGM : le débat impossible (LeMonde.fr : 13/06/2014); L'impossible débat sur le nucléaire (Slate.fr : 25/02/2013); Polémique sur les OGM : «Personne n'ose se confronter aux lobbys » (Sudouest.fr : 23/09/2012); Le nouveau rapport du GIEC relance la polémique sur le réchauffement climatique (LaTribune.fr : 29/09/2013).

\section{RÉSUMÉS}

Cette contribution s'intéresse à certaines modalités de construction de la communication verbale conflictuelle dans les espaces publics. Elle adopte une analyse linguistique du discours pour étudier une controverse sociale à thème environnemental telle qu'elle s'expose dans le quotidien Ouest-France et telle qu'elle contribue à construire - amorcer, nourrir, désamorcer - un conflit qui mobilise la population bretonne sur la question de l'extraction industrielle de sable en baie de 
Lannion. Au terme d'une analyse du discours autour de cette controverse, il est intéressant de relever certaines autres caractéristiques d'un énoncé agonal : (1) il peut être un révélateur de l'existence d'une controverse ; (2) il peut être un révélateur du « climat » de la controverse ; (3) le lexique utilisé pour désigner les acteurs de la polémique, ainsi que la position qui leur est attribuée dans les deux parties de la séquence, peuvent être constitutifs d'une construction linguistique de l'ennemi ; (4) la prise de distance, ou au contraire les marques de proximité entre le locuteur et chacun des deux acteurs, peuvent nous indiquer si un journaliste ou la rédaction d'un journal prend position dans une controverse; (5) de nombreuses autres marques linguistiques d'un énoncé agonal peuvent contribuer à la construction linguistique de l'ennemi (implicite, humour, marques d'atténuation ou de renforcement, paroles rapportées, etc.).

This paper is focused on modalities to build the conflictual verbal communication in the public space. By adopting a linguistic analysis of discourse, it attempts to study an social controversy on the environmental matter, which is featured in a regional French newspaper (Ouest-France). We focus on the ways the journal tends to initiate, feed or de-escalate the conflict which mobilizes part of the Breton people on an industrial project for the sand extraction in the Bay of Lannion.

The conditions for a deliberative space of confrontation for both discourse and counter-discourse were in place in order to build an argumentation, namely a "modality of discursive management for disagreement" (Doury, 2016). An argumentation is not systematically conflictual. However, it appeared in our case that the contradiction in the argumentation was systematically under the form of a stigmatization. When reading the corpus of papers from Ouest-France, we were struck by the very negative image of the industrial company responsible for the sand extraction ( $\mathrm{La}$ Compagnie armoricaine de navigation, or CAN).

Facing such an unbalanced argumentative discourse, characterized by a polarized position (Amossy, 2014) likely to create and to feed conflict, we were interested in the linguistic construction of this negative image of one of the opponents (the CAN). We studied how this construction appeared in the public space, in the management of public affairs. Our analysis focused on such a recurrent discursive process, whihc we named the "agonal statement," characterized by a symptom of conflictual discursive exchanges and, more generally, by a symptom of the conflictual intersubjective relation. The study was based on the notions of dialogism (Bakthine, 1975), as well as the notions of common sense and ideologization (Sarfati, 2004, 2011), in order to examine if the discourse can be used to displace its argumentative equilibrium by the construction of an enemy concentrating the hostile criticisms in the conflictual exchange in the form of monologue.

The agonal statement constitutes the smallest unity containing the linguistic features necessary for dealing with a controversy by writing: actors who oppose each other, and a locutor who reports the event. It is a clue of controversy.

Example: The future Monitoring Committee supposed to reassure ... does not make its effect presently on the people opposed to the [sand] extraction.

Sometimes, the agonal statement is the title of a paper, or its introduction, or one of its subtitles. Sometimes it is the first utterance of an article. Sometimes, it is made of a single utterance without a verb. Therefore, the agonal statement is a relatively simple structure, but its analysis is complex: three features (three socio-ideological actors, three argumentative functions, three utterers) and an interdiscursive context to take into account.

However, such complexity gives rise to important information for the analysis: a simple agonal statement often represents the 'climate' of the whole article, as well as the editorial choices of the locutor and, potentially, the position of the socio-politic actors in the treatment of a public question.

Agonal segments often constitute the beginning of a discourse in giving information on the rationale for the critics and comments which follow. The voice of the first actor of the agonal 
statement is heard only in order to situate the theme of the critics, the condemnation, and the judgment against that actor. In our corpus, the agonal statement is often the first (or one of the first) utterance of a relatively long sequence in which the theses of the opponents against an actor are exposed.

The first actor of an agonal statement is often almost not heard. Moreover, the cotext is often silent concerning his position. The reader won't get more information about his thesis when reading the rest of the paper. And if the cotext is not silent concerning his position, it is very short, or very critical. Conversely, the position of the second actor is often explained in both the agonal statement and the cotext.

In the local daily press, the high frequency of the agonal statement shows that the media discourse keeps the verbal conflict going: it tends to impose a new form of doxa originating from an ideology by a process of ideologization and by giving the preference to one of the voices of the controversy (Sarfati, 2011). This study shows that the analysis of language conflict needs more than a lexical and syntactic approach. It appears that conflict does not proceed by local markers of language violence; it appears through an interdiscursive dialogic process during a discursive moment (Moirand, 2007a). The studied language conflict is indicative of the existence of a prior socio-ideological conflict, but also it is also a means to prevent as much as possible other conflictual expressions, including physical violence.

When performing a critical or a pedagogical discourse analysis, it is often interesting to show other characteristics of an agonal statement: (1) it can be indicative of a controversy; (2) it can be indicative of the 'climate' of a controversy; (3) the lexicon used to name the actors of an agonal statement and their position assigned to the two sides within that sequence, can be constitutive of a process for the linguistic construction of an enemy; (4) the linguistic markers of distance or proximity between the actors can be indicative of whether or not the journalist or the editorial team of the newspaper takes a stance in the controversy; (5) many other linguistic markers in an agonal statement can contribute to the linguistic construction of an enemy (implicit, humor, attenuation or enhancement markers, reported speech).

\section{INDEX}

Keywords : environmental controversy, argumentation, dialogism, ideologization, verbal conflict

Mots-clés : polémique environnementale, argumentation, dialogisme, idéologisation, conflit verbal

\section{AUTEURS}

\section{NATHALIE GARRIC}

Université de Nantes, CoDiRe (EA4643)

nathalie.garric@univ-nantes.fr

\section{MICHEL GOLDBERG}

Université de La Rochelle, LIENSs (UMR 7266, CNRS)

michel.goldberg@univ-lr.fr 